\title{
The Hippocampus and Disambiguation of Overlapping Sequences
}

\author{
Kara L. Agster, ${ }^{*}$ Norbert J. Fortin, ${ }^{*}$ and Howard Eichenbaum \\ Laboratory of Cognitive Neurobiology, Department of Psychology, Boston University, Boston, Massachusetts 02215
}

Recent models of hippocampal function emphasize its potential role in disambiguating sequences of events that compose distinct episodic memories. In this study, rats were trained to distinguish two overlapping sequences of odor choices. The capacity to disambiguate the sequences was measured by the critical odor choice after the overlapping elements of the sequences. When the sequences were presented in rapid alternation, damage to the hippocampus, produced either by infusions of the neurotoxin ibotenic acid or by radiofrequency current, produced a severe deficit, although animals with radiofrequency lesions relearned the task. When the sequences were presented spaced apart and in random order, animals with radiofrequency hippocampal lesions could perform the task. However, they failed when a memory delay was imposed before the critical choice. These findings support the hypothesis that the hippocampus is involved in representing sequences of nonspatial events, particularly when interference between the sequences is high or when animals must remember across a substantial delay preceding items in a current sequence.

Key words: rat; hippocampus; sequence memory; sequence disambiguation; episodic memory; olfactory learning; ibotenic acid; radiofrequency
In humans, the hippocampus is critical to episodic memory, the ability to recall unique personal experiences (Vargha-Khadem et al., 1997). To address whether this function of the hippocampus extends to animals, recent theoretical considerations have focused on the unique temporal, spatial, and contextual components of episodic memories (Gaffan, 1994; Mishkin et al., 1997; Clayton and Dickinson, 1998). Recent computational models have specifically emphasized the potential role of hippocampal circuitry in representing sequences of events that compose the spatial and temporal context of an episode (Levy, 1996; Sohal and Hasselmo, 1998; Wallenstein et al., 1998; Eichenbaum et al., 1999; Lisman, 1999). There is evidence that the hippocampus is important for encoding and retrieving sequential information contained in a unique series of spatial (Kesner and Novak, 1982; Chiba et al., 1994) and nonspatial (Fortin et al., 2002) events. In addition, Levy (1996) proposed that sequence coding by the hippocampus may be especially important when the sequences have overlapping elements through which memory of earlier elements must be remembered to complete each distinct sequence. Sohal and Hasselmo (1998) also modeled the overlapping sequence problem and showed how the dynamics of physiological parameters reflected by the theta rhythm could enhance sequence disambiguation by allowing weak associations that match the target sequence win out during retrieval.

The importance of hippocampal function in spatial memory tasks in which ambiguous spatial cues are prevalent (such as in the water maze and T-maze; see Discussion) is well documented (O'Keefe and Nadel, 1978). However, it is not clear from these studies whether the demand for disambiguation of sequences per se, rather than other aspects of spatial processing, is critical. To

\footnotetext{
Received Dec. 17, 2001; revised April 8, 2002; accepted April 17, 2002.

This work was supported by National Institute of Mental Health (NIMH) Grants MH52090 and MH60450 (H.B.E.) and Nature Sciences and Engineering Research Council of Canada (N.J.F).

*K.L.A. and N.J.F. contributed equally to this work.

Correspondence should be addressed to Dr. Howard Eichenbaum, Department of Psychology, Boston University, 64 Cummington Street, Boston, MA 02215. E-mail: hbe@bu.edu.

Copyright (ㄷ) 2002 Society for Neuroscience $\quad 0270-6474 / 02 / 225760-09 \$ 15.00 / 0$
}

test whether sequence disambiguation is a fundamental feature of memory processing dependent on the hippocampus, we designed a sequence disambiguation task after Levy's (1996) formal model that involved two series of events that overlap in the middle items (see Fig. 1). The sequences were presented as a series of six pair-wise odor choices where, for each sequence, selection of the appropriate odor at each choice point was rewarded. Each trial began with two forced choices that initiated production of one of the two sequences. Then the animal was presented with two forced choices that were the same for both sequences. Subsequently, the subject was allowed a free choice and was rewarded for selecting the odor assigned to the ongoing sequence. Finally, the animal completed that sequence with one more forced choice. The critical feature of this task was the free choice; on that test, animals were required to remember their choices from the first two pairings of the current sequence during the ambiguous components of the trial and then use the earlier information to guide the correct odor selection.

\section{MATERIALS AND METHODS}

\section{Experiment 1: effects of ibotenic acid lesions of the hippocampus on sequence alternation}

In this experiment, the serial events were constituted as two partially overlapping sequences of odor choices, which the rats learned to alternate. In the schematic representation of the sequences shown in Figure 1 , the rats were initially trained to select odor $A$ over odor $L$, then $B$ over $\mathrm{M}$, then $\mathrm{X}$ over $\mathrm{W}$, then $\mathrm{Y}$ over $\mathrm{Z}$, then $\mathrm{E}$ over $\mathrm{P}$, then $\mathrm{F}$ over $\mathrm{Q}$. After successful acquisition of sequence 1 , they were trained on sequence 2 , in which they were rewarded for making the opposite selection in each pairing, with the exception of pairs involving $\mathrm{X}$ and $\mathrm{Y}$ where the correct choices remained constant. Animals were retrained on each sequence separately and then presented with the two sequences repetitively in alternation. After surgery for hippocampal ablation or a sham lesion, animals were again tested on the sequence alternation problem.

\section{Subjects}

Eight male Long-Evans rats served as subjects. The animals were experimentally naive and weighed $\sim 225-250 \mathrm{gm}$ at the beginning of the experiment. They were housed individually in plastic cages $(47.5 \mathrm{~cm}$ long $\times 26 \mathrm{~cm}$ wide $\times 21 \mathrm{~cm}$ high) and placed on a $12 \mathrm{hr}$ light/dark cycle, with all testing occurring during the light phase. Animals were kept 
a.

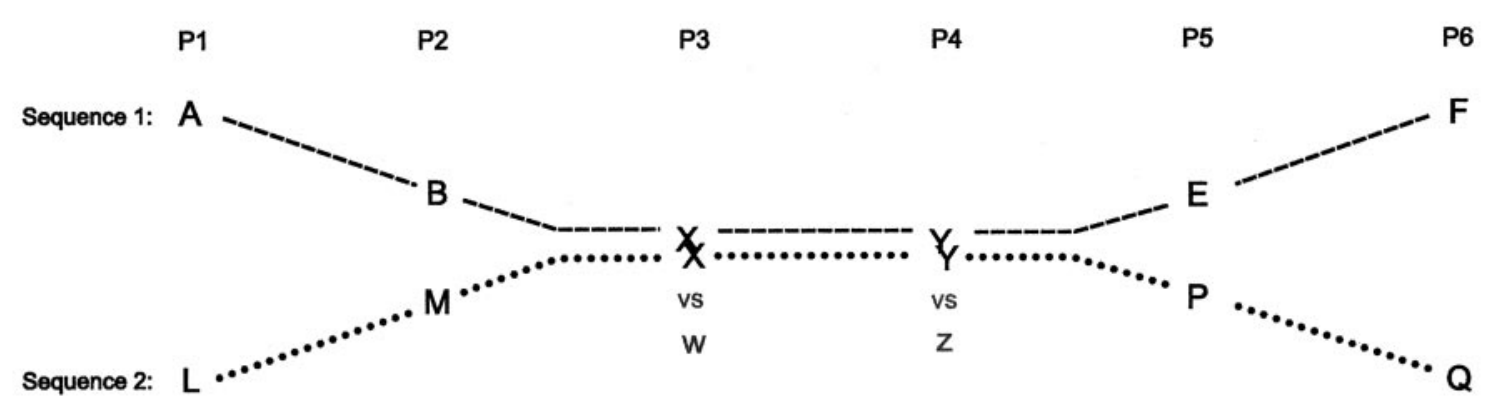

b.

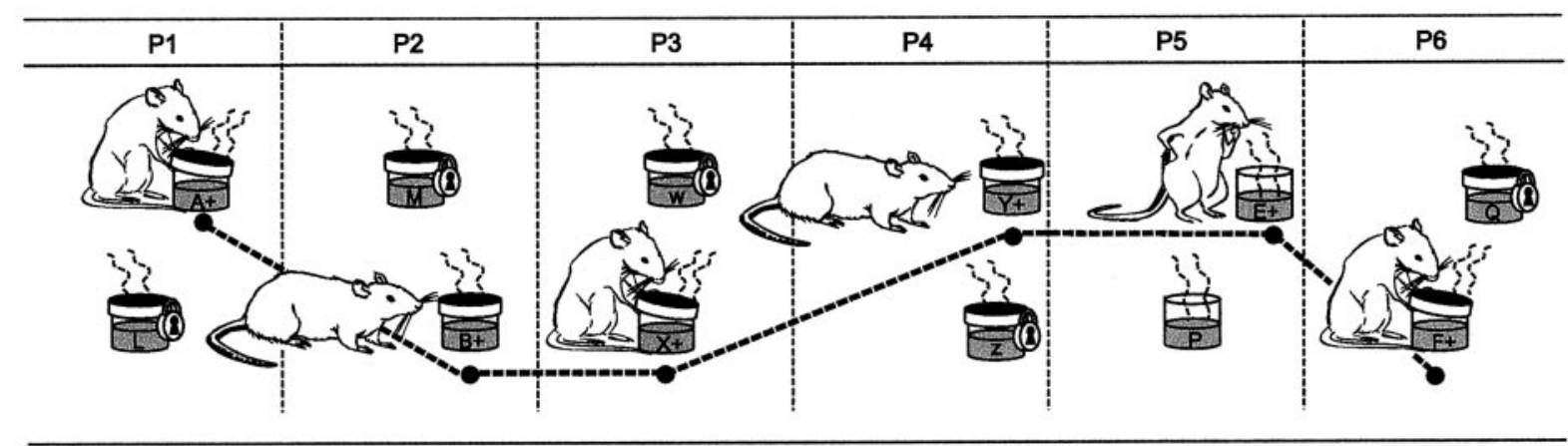

Figure 1. The odor sequence task. $a$, The two odor sequences are indicated by letters. In performing each sequence, the rat selected between vertically aligned odors in each sequence; in both sequences, $X$ was to be selected over $W$, and $Y$ over $Z$. $b$, Illustration of an example trial on sequence 1 . The location where the odors are presented is randomly determined. On the first four pairs $(P 1-P 4)$ and pair $6(P 6)$, the animal was required to lift the perforated lid and dig from the cup containing the reward; the lid of the alternative choices was "locked." On pair 5 (P5), no lids are used, and the first choice is scored. + , Reinforced odor.

mildly food-deprived to maintain at least $85 \%$ of free-feeding body weight with ad libitum access to water throughout testing. Animals were trained $5 \mathrm{~d}$ each week.

\section{Apparatus and materials}

Training and testing were conducted in the home cage. During testing the cage was surrounded by three $51-\mathrm{cm}$-high translucent acrylic walls, one at the back and one on either side, to prevent the animals from leaving the cage during the trials. An acrylic platform with a wire handle was used to lower two stimulus cups at the front end of the cage. Translucent Nalgene cups measuring $70 \mathrm{~mm}$ in diameter and $63 \mathrm{~mm}$ in height were used. Each cup was filled with $99.2 \mathrm{gm}$ of playground sand mixed with $0.8 \mathrm{gm}$ of a ground household spice. The spices were as follows: $\mathrm{A}=$ allspice, $\mathrm{B}=$ dill, $\mathrm{X}=$ paprika, $\mathrm{Y}=$ nutmeg, $\mathrm{E}=$ lemon, $\mathrm{F}=$ celery, $\mathrm{L}=$ tarragon, $\mathrm{M}=$ cinnamon, $\mathrm{P}=$ mint, $\mathrm{Q}=$ fennel, $\mathrm{W}=$ basil, and $\mathrm{Z}=$ coffee. On some presentations (see below) the cups were covered with lids in which 18 holes of $9 \mathrm{~mm}$ diameter were drilled, allowing the rat to smell the odorized sand inside. The lids were all identical in appearance. However, in some cases the lids were screwed on ("locked"), preventing opening of the cup; in other cases the threads were shaved, allowing the "unlocked" lid to be removed easily.

\section{Training protocol}

Shaping. Animals were handled for $3 \mathrm{~d}$ before training. Rats were allowed to approach and dig in a cup of unscented sand to obtain a buried cereal reward (Froot Loops, Kellogg's, Battle Creek MI). Then the animals were shaped by successive approximations to remove a lid placed on top of the cup to uncover the sand. Sand scented with various odors was then introduced, and training began when the animals were habituated to the presence of scented sand.

Sequence training. Animals were initially trained on each sequence individually, to encourage formation of a distinct representation for each series of choices. Rats were first required to select the appropriate odors corresponding to sequence 1 (Seq1) (Fig. 1). Each trial was composed as a series of pair-wise odor choices. At the outset of each choice, an opaque acrylic plaque was used to push the animal to the area opposite the location of the cup presentation. The platform containing the cups was lowered to one of five randomly selected standard positions at the front end of the cage: parallel to the front cage wall and centered; parallel to the front wall but displaced to the left or right of the cage; or in either corner forming a $45^{\circ}$ angle between the front and side walls. Also, the left-right arrangement of the cups on the platform was varied pseudorandomly, such that the rewarded cup could appear in any of 10 possible locations across trials. After positioning of the platform, the animal was allowed to slowly approach and sniff the cups. The animal was considered to have made a response at the moment a paw touched the sand in one of the cups. The interval between presentations was $15 \mathrm{sec}$, and the intertrial interval was 3 min.

On the initial three trials of training on Seq1, each of the animals' six choices was guided using a locked lid on the incorrect choice and an unlocked lid on the correct choice, thereby preventing the animals from digging in the unreinforced cup. For trials four through six, no lids were used on any of the choices, and animals were allowed to correct themselves on each choice. On all subsequent trials, no lids were used, and no corrections were permitted on any choice. Rats continued training with three to six trials per day until they reached a criterion of four or fewer cumulative errors across four consecutive sequences. Subsequently, the animals were trained on Seq2 (Fig. 1) following the same protocol. After reaching the criterion, they were then retrained on Seq1, and then retrained on Seq2, such that they reached the criterion for each sequence twice before proceeding to the preoperative testing phase.

Training on sequence alternation. In this phase, animals were trained for six trials per session presented in strict alternation (i.e., Seq1-Seq2-Seq1Seq2-Seq1-Seq2, or Seq2-Seq1-Seq2-Seq1-Seq2-Seq1). The interval between choice presentations was $15 \mathrm{sec}$, and the intertrial interval was 1 min. During the initial 30 trials, to accommodate animals to the appearance of lids on some choices and not on others, lids were used on all but one randomly selected pair in each sequence. In subsequent training we were particularly interested in performance on the first choice after the ambiguous components of the sequence. Therefore, to avoid any possible confounding influence of errors that could occur on earlier choices in the trial, locked and unlocked lids were used to direct choices to the correct cup on pairs 1-4 (P1-P4) as well as pair 6 (P6). On P5 no lids were used, allowing the rat to freely express its knowledge of the correct choice in the current sequence. Rats were tested on this phase until they attained a criterion of at least $75 \%$ correct on P5 choices over five sessions. For 


\begin{tabular}{llll}
\hline $\begin{array}{l}\text { Table 1. Sites and injection volumes used in making ibotenic acid } \mathbf{( 1 0} \\
\boldsymbol{\mu g} / \boldsymbol{\mu l}) \text { lesions }\end{array}$ & & & \\
& & & $\begin{array}{l}\text { Injection volume } \\
(\mu \mathrm{l})\end{array}$ \\
Anteroposterior & Mediolateral & Dorsoventral & 0.22 \\
\hline-2.2 & \pm 1.0 & -3.2 & 0.1 \\
-3.0 & \pm 1.4 & -3.4 & 0.1 \\
& & -2.8 & 0.2 \\
& \pm 3.0 & -3.0 & 0.1 \\
-4.0 & \pm 2.1 & -3.3 & 0.1 \\
& & -2.3 & 0.2 \\
-4.8 & \pm 3.7 & -3.0 & 0.2 \\
& \pm 5.1 & -7.0 & 0.2 \\
-5.7 & \pm 4.1 & -7.2 & 0.1 \\
& & -3.5 & 0.2 \\
& \pm 4.5 & -3.6 & 0.1 \\
& \pm 5.1 & -6.8 & 0.14 \\
& & -5.5 & 0.14 \\
\hline
\end{tabular}

each choice presentation, the animals were pushed back in the cage area opposite the cups and allowed only one response before the cups were removed. Animals were rewarded with one-fourth of a Froot Loop on P1-P4 and P6; on P5 a whole Froot Loop was the reward.

\section{Surgery}

Pairs of animals were matched for trials to reach the criterion and then randomly assigned to either the control or the hippocampal lesion group. The rats in the hippocampal group were anesthetized with $1 \%$ halothane and placed in the stereotaxic apparatus after their heads had been shaved. They were injected with $0.081 \mathrm{mg}$ of atropine sulfate to prevent respiratory difficulties, and their body temperature was maintained with a heating pad. The skin was incised along the midline, the skull was exposed up to the lateral ridges, and a section of skull corresponding to the area of the hippocampus was removed bilaterally. At each injection site (Table 1), the dura was pierced using a small syringe tip, and the microsyringe (Hamilton, Reno, NV) was lowered into the brain. One minute was allowed for the brain to settle and then a variable volume of ibotenic acid (Table 1) was injected over a period of 1 min using a microinjector unit (Kopf Instruments, Tujunga, CA). A waiting period of 1 min allowed for diff usion of the ibotenate, after which the microsyringe was raised. The microsyringe was cleaned and inspected for obstructions between each injection.

Subsequently, the wound was sutured and covered with a topical antibiotic. Animals then received a systemic injection of antibiotic (250 $\mathrm{mg} / \mathrm{kg}$; Cefazolin, Bristol-Myers Squibb, Princeton, NJ), and $5 \mathrm{ml}$ of saline/dextrose solution was injected subcutaneously to facilitate recovery. Control subjects underwent the same procedure except that the microsyringe was not lowered into the brain. Testing resumed after a 2 week recovery period.

\section{Testing after surgical recovery}

Animals were tested on the alternation task for 10 six-trial postoperative sessions, during which P5 was presented without lids. For comparison, we subsequently tested the animals on 10 additional sessions during which lids were used on all choices except P6. Note that the animals were not tested on P6 preoperatively. We expected that the demand for sequence disambiguation would be diminished in this protocol because a correct choice on P6 could be guided by the information provided in the immediately preceding choice (P5).

\section{Histology}

Animals were overdosed with sodium pentobarbital and perfused transcardially with saline, followed by a $10 \%$ formalin solution. Brains were removed, kept in formalin for $1 \mathrm{~d}$, and stored in a $30 \%$ glycerin solution overnight for cryoprotection. Brains were sectioned at $50 \mu \mathrm{m}$ using a
Table 2. Sites and injection volumes used in making radiofrequency lesions

\begin{tabular}{lllc} 
Anteroposterior & Mediolateral & Dorsoventral & $\begin{array}{l}\text { Current } \\
(\mathrm{mA})\end{array}$ \\
\hline-2.2 & \pm 1.0 & -3.5 & 8 \\
-3.2 & \pm 1.4 & -3.1 & 8 \\
& \pm 3.0 & -3.0 & 8 \\
-4.0 & \pm 2.5 & -3.2 & 8 \\
& \pm 3.7 & -3.1 & 8 \\
-4.8 & \pm 4.9 & -6.6 & 9 \\
& & -5.4 & 8 \\
& \pm 4.3 & -6.9 & 9 \\
-5.4 & & -3.5 & 9 \\
& \pm 4.2 & -3.6 & 9 \\
& \pm 5.0 & -6.6 & 9 \\
& & -5.5 & 11
\end{tabular}

microtome, with every fourth slice mounted on slides, and later stained with cresyl violet for examination of lesion size and placement.

\section{Experiment 2: effects of radiofrequency lesions of the hippocampus on sequence alternation}

The results of experiment 1 suggested that the hippocampus is critical for disambiguating overlapping sequences, and that there appears to be little improvement in performance of hippocampal rats over 10 sessions of continued training. However, ibotenic acid injections were used to damage the hippocampus in that experiment. Ibotenate is an excitotoxin that kills neurons by causing them to fire at abnormally high rates until death, and it is clear that this abnormal activity has the potential for propagation of cellular damage to other brain regions. This technique sometimes leads to significant incidental damage to neurons in other brain areas, including the entorhinal cortex and the thalamus, caused by propagation of abnormal activity and leakage during infusion (Anagnostaras et al., 2002). Such incidental neuronal loss could affect performance on sequence disambiguation, because damage to cortical areas neighboring the hippocampus is known to exacerbate the effects of hippocampal damage (Zola-Morgan et al., 1993, 1994). Therefore, we replicated experiment 1 using radiofrequency (RF) current to damage the hippocampus. This method does not distinguish neurons and fibers of passage and instead destroys tissue locally by heat. Thus, radiofrequency lesions avoid the potential for widespread cell damage caused by the propagation of aberrant electrical activity, as well as the potential for extrahippocampal damage resulting from leakage of the neurotoxin outside the target lesion site.

Subjects. Subjects were 10 behaviorally naive, male Long-Evans rats. Their body weight at the beginning of the experiment was between 225 and $250 \mathrm{gm}$. Rats were housed, fed, and watered as described in experiment 1.

Training protocol. The shaping and initial training protocol were the same as in experiment 1, except that locked and unlocked lids were used on P1-P4 and P6 from the outset of alternation training. The procedures in sequence alternation testing were identical to those of experiment 1 . The spices were as follows: $\mathrm{A}=$ parsley, $\mathrm{B}=$ dill, $\mathrm{X}=$ orange, $\mathrm{Y}=$ basil, $\mathrm{E}=$ lemon, $\mathrm{F}=$ paprika, $\mathrm{L}=$ allspice, $\mathrm{M}=$ tarragon, $\mathrm{P}=$ celery, $\mathrm{Q}=$ fennel, $\mathrm{W}=$ cocoa, and $\mathrm{Z}=$ nutmeg.

Surgery. Pairs of animals were matched for preoperative performance levels and then randomly assigned to either the control or the hippocampal lesion group. The same surgical procedures as in experiment 1 were used, with the exception that a Radionics RFG-4A (Burlington, MA) was used to generate radiofrequency lesions in the hippocampus. The electrode was an insulated $100 \mu \mathrm{m}$ nichrome wire with $0.7 \mathrm{~mm}$ of the insulation removed at the tip. At each of 12 sites bilaterally (Table 2), dura was pierced using a small syringe tip, and the electrode was then lowered into the brain. One minute was allowed for the brain to settle, then a variable amount of current (Table 2) was passed for another minute, and finally the electrode was removed after a 1 min waiting period. Sham controls underwent the same surgical treatment, except that the electrode was not lowered into the brain after dura was punc- 
Experiment 1 (IBO)
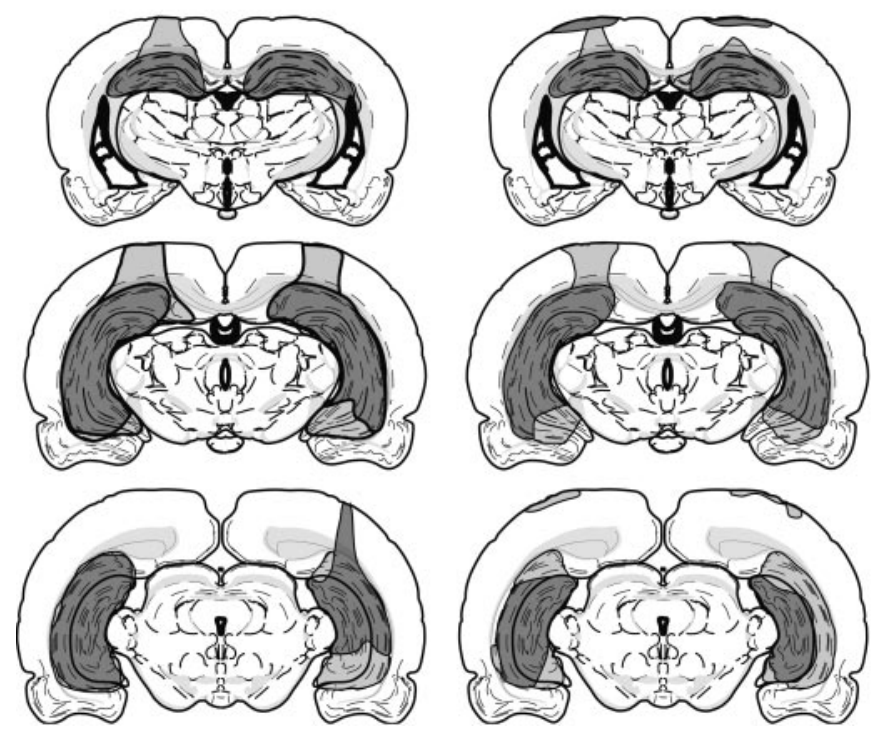

tured. Post hoc histological procedures and analyses were identical to those performed in experiment 1 .

\section{Experiment 3: effects of hippocampal lesions on disambiguation of sequences presented in random order}

The results of experiment 2 indicate that restricted lesions of the hippocampus produced by radiofrequency current result in a substantial impairment in sequence disambiguation, but these animals eventually acquired the task. In addition, the sequence alternation task could have been solved in either of two ways. Animals could be making their selection on the critical pair 5 on the basis of a representation of previous items within the current sequence. Alternatively, they could be simply alternating between trials the item selected on the critical pair 5, without reference to previous items in the current sequence. To investigate whether there is a lasting impairment after restricted radiofrequency hippocampal lesions on a task where sequence disambiguation requires reference to previous items within the current sequence, an additional experiment involved testing animals with radiofrequency lesions on disambiguation of sequences represented in random order.

Subjects. Subjects were nine behaviorally naive, male Long-Evans rats. Their body weight at the beginning of the experiment was between 225 and $250 \mathrm{gm}$. Rats were housed, fed, and watered as described in experiments 1 and 2.

Training protocol. The shaping and initial training protocol were the same as in experiment 2, except that animals were pretrained in daily four-trial sessions to a criterion of four or fewer cumulative errors across four consecutive sequences. Sequence 1 was $\mathrm{A}=$ coffee, $\mathrm{B}=$ anise, $\mathrm{X}=$ marjoram, $\mathrm{Y}=$ parsley, $\mathrm{E}=$ orange, and $\mathrm{F}=$ cocoa; sequence 2 was $\mathrm{L}=$ cinnamon, $\mathrm{M}=$ dill, $\mathrm{X}=$ marjoram, $\mathrm{Y}=$ parsley, $\mathrm{O}=$ basil, and $\mathrm{P}=$ mint, $\mathrm{W}=$ celery, $\mathrm{Z}=$ thyme; each $0.8 \%$ by weight. Pretraining to the criterion on each independent sequence was repeated twice in alternation. Subsequently, the procedures in sequence disambiguation training and testing were identical to those of experiment 2, except that the interval between presentation of the sequential choices was $30 \mathrm{sec}-1 \mathrm{~min}$, the intertrial interval was $15 \mathrm{~min}$, and the sequences were presented in random order such that each session consisted of three trials on each sequence. Rats were trained preoperatively at this stage to a criterion of $75 \%$ correct on pair 5 choices over five sessions. They were tested postoperatively for 10 sessions with a minimal delay between choices, and then for an additional 8 sessions with a delay of $30 \mathrm{~min}$ introduced between the fourth and fifth choices on each trial.

Surgery. Pairs of animals were matched for preoperative performance levels and then randomly assigned to either the control or the hippocampal lesion group. The surgical procedures, recovery, and histological analyses were the same as in experiment 2 .

\section{Experiment $3(\mathrm{RF})$}

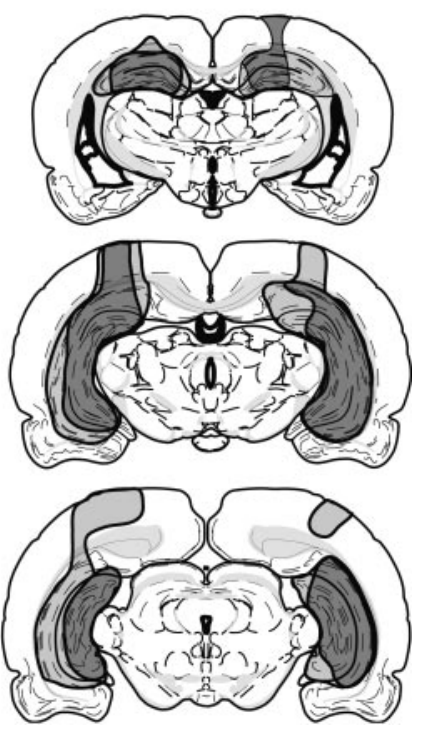

Figure 2. Reconstructions of the smallest and largest hippocampal lesions. Coronal sections are adapted from Swanson (1992). Light gray refers to the largest volume of hippocampus damaged; dark gray indicates smallest lesion.

\section{RESULTS}

\section{Experiment 1}

The goals of this experiment were to determine whether rats can learn to alternate two partially ambiguous sequences of odor choices and to ascertain whether performance on this task depends on hippocampal function. The following sections describe the performance of rats in learning ambiguous odor sequences and sequence alternation and the effects of selective neurotoxic lesions of the hippocampus on alternation of ambiguous sequences. Our analyses focused on characterizing the rate of learning by normal rats in this task and on comparing performance between control and hippocampal rats on the critical choice after the ambiguous component in the sequences (pair 5) and, for comparison, on the subsequent choice (pair 6) that did not require memory through the ambiguous segment.

\section{Training}

During the initial sequence training, animals learned the first presentation of Seq1 in 21.6 trials (SD, 7.0; range, 17-33), the first presentation of Seq2 in 21.1 trials (SD, 6.4; range, 10-28), the second presentation of Seq1 in 13.4 trials (SD, 5.1; range, 7-24), and the second presentation of Seq2 in 13.0 trials (SD, 4.9; range, 7-22). Two rats required many more trials than the others and were therefore trained again on Seq1, then Seq2, learning each in 7 and 22 trials and 9 and 7 trials, respectively. In the subsequent sequence alternation test phase, rats reached the criterion of $75 \%$ correct over 30 consecutive trials in an average of 277.8 trials (SD, 49.8; range, 240-354). Two animals did not reach the preoperative criterion; one animal did not succeed after 420 trials, and the other ceased performing choices after 342 trials. The total period of preoperative training was $23-28$ weeks.

\section{Hippocampal lesions}

Injection of ibotenic acid produced a complete loss of cells in all subfields of the hippocampus proper (Fig. 2). We intentionally avoided the subiculum to avoid causing inadvertent damage to surrounding areas, including the entorhinal cortex. However, in one animal the anterior part of the dorsal subicular region was 


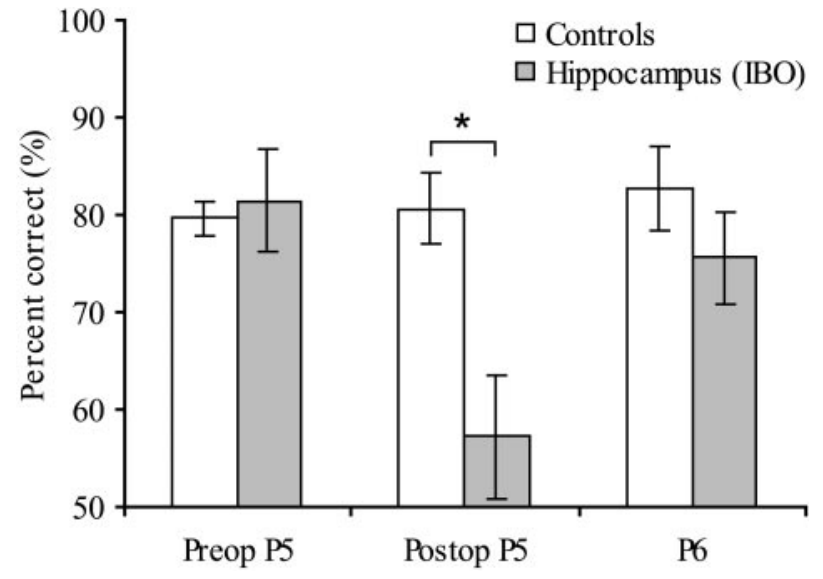

Figure 3. Performance (mean $\pm \mathrm{SE}$ ) of control rats and rats with ibotenic acid $(I B O)$ hippocampal lesions on pair $5(P 5)$ and pair $6(P 6)$ tests. ${ }^{*} p<0.025$.

damaged bilaterally, and another animal showed very slight damage to the ventral subiculum $(<10 \%)$ and medial entorhinal cortex $(<5 \%)$. Two animals also had bilateral damage to the cortex immediately overlying the hippocampus. We estimated the extent of the lesion at three anteroposterior coordinates $(-3.90$ $\mathrm{mm},-5.25 \mathrm{~mm}$, and $-6.06 \mathrm{~mm}$ ). Damage to the hippocampal subfields and dentate gyrus ranged from 75 to $98 \%$ of total volume. These estimates are lower (68-94\%) when the subiculum is included in computing the total volume of hippocampal damage.

\section{Performance after surgery}

After surgical recovery, choice performance on the fifth (P5) and sixth (P6) odor pairs was compared between groups and with performance on P5 on the last 10 preoperative sessions (on those sessions, animals always completed six trials per session). Control rats maintained a high and equivalent level of performance across conditions (Fig. 3). By contrast, the rats with damage to the hippocampus performed poorly on P5 but well in subsequent testing on P6. A repeated-measures ANOVA confirmed that the groups performed differently among these phases (significant group $\times$ phase interaction; $\left.F_{(2,8)}=9.278 ; p=0.0082\right)$. Post hoc analyses were conducted as simple main effects of group at a specific phase. These analyses used a pooled error term based on the variability of the groups across all levels of phase and were evaluated at $\alpha^{\prime}=\alpha / 2$ because two post hoc comparisons were made (Kirk, 1982; Girden, 1992). These tests showed that performance between the two groups differed significantly after surgery only on P5 ( $\left.F_{\text {Group at Postop P5(1,4) }}=12.90 ; p<0.025\right)$. Furthermore, separate $t$ tests showed that the performance of hippocampal rats on P5 was not significantly above chance $t_{(2)}=$ $1.127 ; p=0.3769)$, whereas performance of the same animals was above chance before surgery on P5 $\left(t_{(2)}=6.109 ; p=0.0258\right)$ as well as on P6 after surgery $\left(t_{(2)}=5.274 ; p=0.0341\right)$. The performance of control subjects was above chance on all testing phases (before surgery P5: $t_{(2)}=16.016, p=0.0039$; after surgery P5: $t_{(2)}=8.385, p=0.0139 ;$ P6: $\left.t_{(2)}=7.554, p=0.0171\right)$.

It was unclear whether the success of the hippocampal rats on P6 was caused by the diminished cognitive demands on this pairing or whether the extended testing experience led to a recovery of function. To investigate whether there was improvement over the course of sessions on P5, we compared postoper-

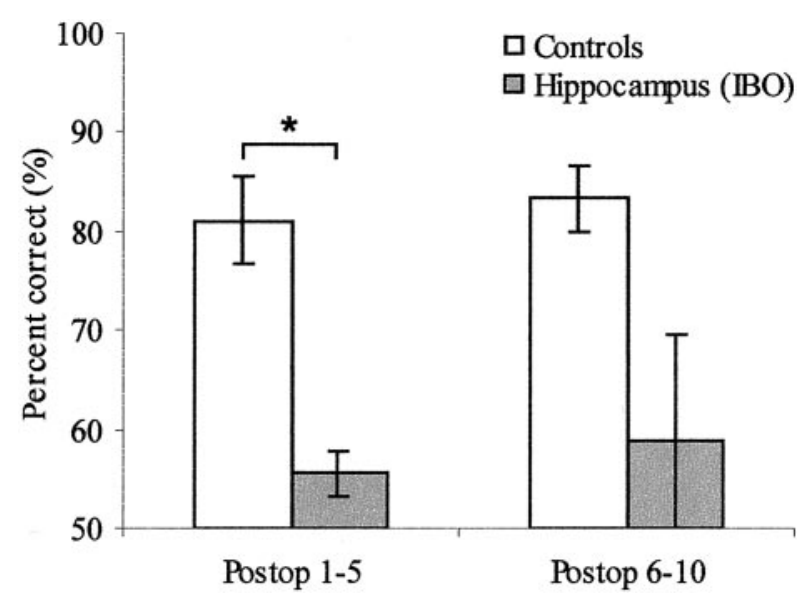

Figure 4. Performance (mean $\pm \mathrm{SE}$ ) on pair 5 judgements by control rats and rats with ibotenic acid (IBO) hippocampal lesions on the first and second five-session blocks of postoperative sessions. * $p<0.025$.

ative performance on P5 between the first and second postoperative blocks of five sessions (Fig. 4). Repeated-measures ANOVA confirmed the deficit (significant group $\times$ block effect; $F_{(2,8)}=$ $7.910 ; p=0.0127$ ), and post hoc tests showed that the performance between the two groups differed statistically only on the first postoperative block ( $F_{\text {Group at Postop 1-5(1,4) }}=26.28 ; p<$ $0.025)$. The difference between the groups on the second block failed to reach significance $\left(F_{\text {Group at Postop } 6-10(1,4)}=4.84 ; p>\right.$ $0.025)$, although on that block the performance of the controls was significantly better than chance $\left(t_{(2)}=10.0 ; p=0.0099\right)$, whereas the performance of the lesioned rats was not $\left(t_{(2)}=\right.$ $0.838 ; p=0.4901)$. In addition, the performance of the hippocampal rats did not differ between the two blocks $\left(F_{\text {Hipp at Postop 1-5 vs Hipp at Postop } 6-10(1,4)}=9.235 ; p>0.0166 ; \alpha / 3\right.$ was used because three post hoc comparisons were calculated). This result suggests that the success of hippocampal rats on P6 is likely not attributable entirely to a recovery of function, but rather to the decrease in cognitive demands associated with that problem.

These results indicate that immediately after surgical recovery, animals with damage to the hippocampus are severely impaired in their capacity to disambiguate overlapping sequences, and only slight improvement was apparent with continued testing. The observation of a deficit in the critical free choice on pair 5 suggests that animals with hippocampal damage cannot maintain a representation of the initially chosen items in the current sequence through a set of ambiguous choices. However, with additional training, an alternative strategy that partially mediates sequence alternation emerges.

\section{Experiment 2}

The goal of this experiment was to evaluate the effects of selective radiofrequency lesions of the hippocampus on alternation of ambiguous odor sequences and to compare the effects of radiofrequency lesions with those of neurotoxic lesions of the hippocampus reported above.

\section{Training}

Rats learned the first presentation of Seq1 in 9.2 trials (SD, 2.4; range, 7-15), the first presentation of Seq2 in 12 trials (SD, 3.5; range, 7-18), the second presentation of Seq1 in 9.5 trials (SD, 3.3; range, 7-14), and the second presentation of Seq 2 in 8.3 trials 


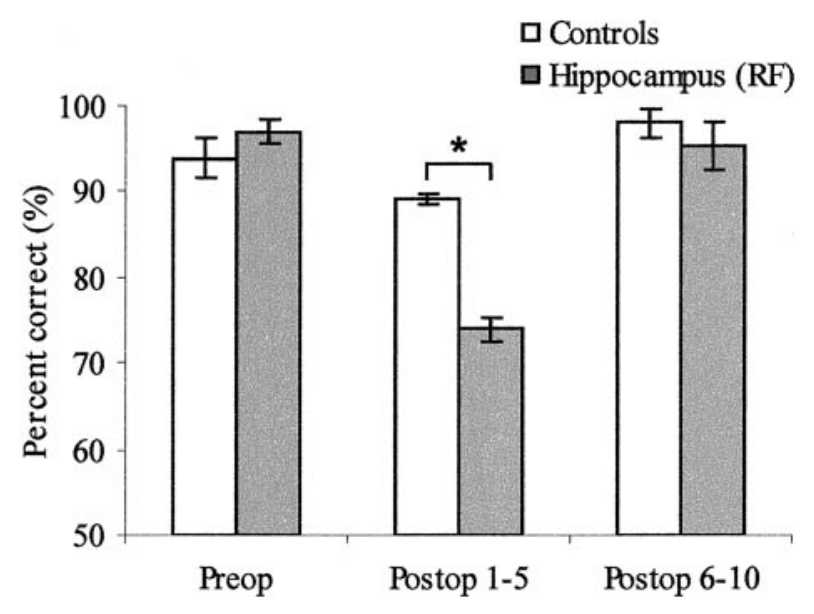

Figure 5. Performance (mean $\pm \mathrm{SE}$ ) of control rats and rats with radiofrequency $(R F)$ hippocampal lesions on preoperative and postoperative pair 5 testing. The postoperative performance is divided into two blocks of five sessions. ${ }^{*} p<0.025$.

(SD, 1.9; range, 7-11). This group of rats learned the sequences in significantly fewer trials than the group tested in experiment 1 $\left(t_{(6)}=3.02, p=0.024\right)$, although there were no differences in the training procedures.

During subsequent sequence alternation testing, animals completed six trials every session. Rats performed at $78.2 \%$ correct on P5 on the first block of five sessions (SD, 5.5; range, 70-86.7\%) and at $95.5 \%$ correct on the second block (SD, 4.3; range, 88.3$100 \%)$. The total period of preoperative training was 10-13 weeks. Rats in this experiment thus acquired the alternation task much more rapidly than those in experiment 1 , and the differences in training protocol (see above) might underlie this disparity.

\section{Hippocampal lesions}

The volume of hippocampal damage ranged from 74 to $96 \%$ total volume (67-88\% if the subiculum is included) (Fig. 2). All rats suffered minor damage to the cortex overlying the hippocampus (three bilateral, two unilateral). Four animals showed some minor damage to the ventral subiculum bilaterally but only in its most posterior extent, and three of them had minor damage to the dorsal part of the medial entorhinal cortex $(<5 \%$ of total medial entorhinal cortex volume).

\section{Performance after surgery}

Control rats maintained a high and equivalent level of performance throughout testing. By contrast, the rats with damage to the hippocampus were impaired on the first five sessions of testing on P5, but performed as well as controls in subsequent testing on P5 as well as on P6 (Fig. 5). A repeated-measures ANOVA confirmed that the groups performed differently among the testing phases (significant group $\times$ block interaction; $F_{(2,16)}=16.637$; $p=0.0001)$. Post hoc analyses revealed that group performances were statistically different only during the first block on P5 after surgery $\left(F_{\text {Group at Postop } 1-5(1,8)}=36.56 ; p<0.025\right)$. Both groups nevertheless performed well above chance on all conditions (Controls: before surgery P5: $t_{(4)}=18.818, p<0.0001$; after surgery P5 Block 1: $t_{(4)}=20.271, p<0.0001$; Block 2: $t_{(4)}=59.406, p<$ 0.0001; Hippocampal: before surgery P5: $t_{(4)}=35.139, p<$ 0.0001; after surgery P5 Block $1: t_{(4)}=14.666, p<0.0001$; Block $\left.2: t_{(4)}=20.094, p<0.0001\right)$. Performance on P6 after surgery was
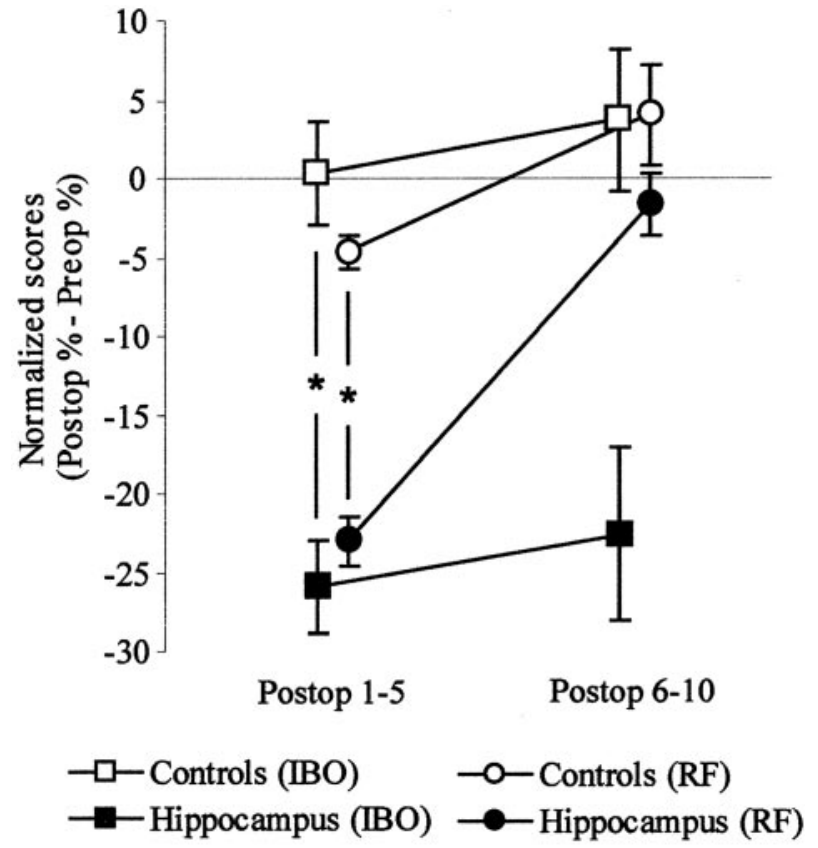

Figure 6. Performance (mean $\pm \mathrm{SE}$ ) of control rats and rats with ibotenic acid $(I B O)$ or radiofrequency $(R F)$ hippocampal lesions on postoperative pair 5 choices. Data are shown in two blocks of five sessions. The normalized scores were obtained by subtracting the preoperative performance level from the performance on each postoperative block. Note that a negative score indicates a decrease in performance from the preoperative level. ${ }^{*} p<0.025$.

equivalent in the control (mean $=85.3 \%$, SEM 1.7) and hippocampal $($ mean $=86.7 \%$, SEM 2.8) groups.

\section{Comparison of the effects of ibotenate and radiofrequency lesions}

To directly compare the magnitude and pattern of the deficits observed after IBO and RF lesions of the hippocampus, we first normalized the individual scores by calculating the difference between P5 performance before surgery and P5 performance on the first and second blocks of five postoperative sessions and then compared the findings in the two experiments (Fig. 6). In both experiments, control animals performed as well as they did preoperatively throughout postoperative testing. In contrast, IBO and RF rats were impaired to a similar degree in the first block of postoperative testing, as indicated by a $25.9 \%$ drop and a $23.0 \%$ drop, respectively, in normalized scores. In the second block of postoperative testing, IBO rats continued to be impaired, whereas RF rats showed substantial recovery. A three-way (experiment $\times$ group $\times$ block) repeated-measures ANOVA revealed a significant overall effect of hippocampal lesions $\left(F_{(1,12)}=\right.$ 124.076; $p<0.0001)$, as well as significant differences between the experiments $\left(F_{(1,12)}=7.765 ; p=0.0164\right)$ and performance between test blocks $\left(F_{(1,12)}=15.150, p=0.0021\right)$. Subsequent two-way (experiment $\times$ block) ANOVAs were used to separately compare the performance patterns of controls between the two experiments and to compare the deficits resulting from different types of hippocampal lesions. The comparison of the controls showed that the normalization effectively removed overall performance differences between the two experiments (main effect of experiment: $\left.F_{(1,6)}=0.638 ; p=0.4548\right)$. Furthermore, performance in the controls did not differ across postoperative testing blocks (main effect of block: $F_{(1,6)}=3.752 ; p=0.1009$ ). The 
separate analysis of performance by the two hippocampal groups revealed that they differed in severity of impairment (main effect of experiment: $\left.F_{(1,6)}=44.269, p=0.0006\right)$, that performance on the first postoperative block of P5 was more severely impaired than on the second block (main effect of block: $F_{(1,6)}=12.086$, $p=0.0132$ ), and that the two groups performed differently between the two postoperative blocks (experiment $\times$ block interaction: $\left.F_{(1,6)}=6.445, p=0.0442\right)$. Post hoc analyses showed that the performance of IBO- and RF-lesioned rats was equivalent on the first postoperative block $\left(F_{\text {Experiment at Postop 1-5(1,4) }}=\right.$ $0.78 ; p>0.025)$, but the groups differed in performance on the second block $\left(F_{\text {Experiment at Postop } 6-10(1,4)}=15.11 ; p<0.025\right)$. These findings indicate that the deficits observed after IBO and RF lesions are initially similar in their magnitude but the ibotenic acid lesions produce a more lasting deficit, whereas rats with RF lesions recover from their initial impairment. It is important to note that the duration of training was greater in the IBO rats than that in RF rats, which might have allowed for a greater extent of consolidation of the initial learning in the IBO rats. This would be expected to result in a less severe impairment in IBO rats than RF rats, contrary to our observations. Therefore it seems unlikely that the differences in training protocols leading to differential opportunity for presurgical consolidation could account for the distinctions in IBO and RF lesions.

\section{Experiment 3}

The goals of this experiment were to evaluate the effects of selective radiofrequency lesions of the hippocampus on disambiguation of overlapping odor sequences that are presented in random order and compare performance when the animal executes its choices in rapid succession with that when a memory delay is imposed following the ambiguous components of the sequence. Three animals were excluded from our analyses: one that did not recover from surgery, one that developed an unrelated skin disease, and one that had substantial damage outside the hippocampus (see below).

\section{Training}

Preoperatively, rats required on average 12.5 trials to learn the first presentation of Seq1 (SD, 1.8974; range, 10-14), 12.6 trials for the first presentation of Seq2 (SD, 2.9136; range, 7-17), 7.5 trials for the second Seq1 (SD, 1.081; range, 7-10), and 7.4 trials for the second Seq2 (SD, 1.2649; range, 7-11). After 10 sessions of random-order presentations of the sequences (six trials each), subjects averaged $88.9 \%$ correct choices on pair 5 (range, 83.3$96.7 \%$ ). Subjects were then divided into two groups that were matched for final performance levels (Fig. 7).

\section{Hippocampal lesions}

The extent of damage for one animal included a substantial portion of the medial entorhinal cortex, and this animal was not considered in the behavioral analyses. The volume of hippocampal damage for the remaining animals ranged from 59 to $82 \%$ total volume (65 to $92 \%$ if the subiculum is included) (Fig. 2). All rats suffered minor damage to the cortex overlying the hippocampus (one bilateral, two unilateral). Every animal showed some minor damage to the ventral subiculum bilaterally but only in its most posterior extent, and one of them had minute unilateral damage to the dorsal part of the medial entorhinal cortex $(<5 \%$ of total medial entorhinal cortex volume).

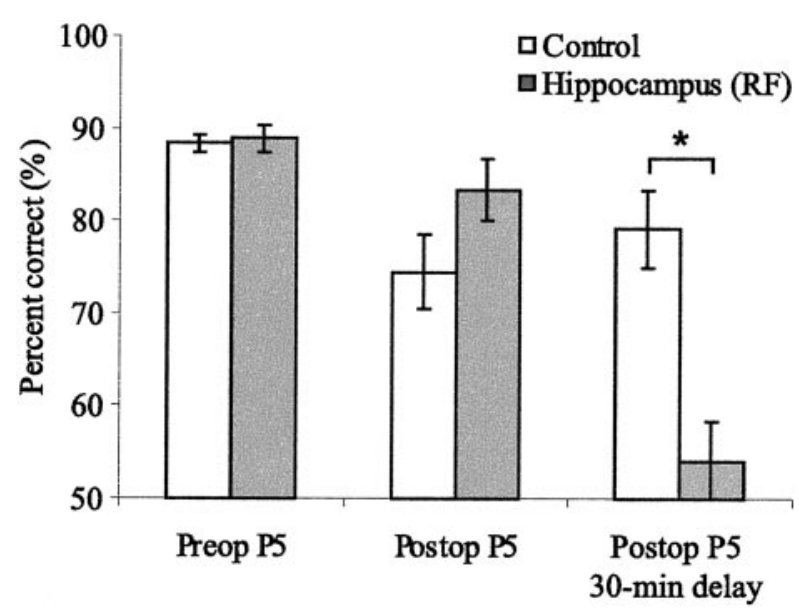

Figure 7. Performance (mean $\pm \mathrm{SE}$ ) of control rats and rats with radiofrequency hippocampal lesions on preoperative and postoperative pair 5 choices in the random-presentation version of the sequence disambiguation task. Scores are shown for the final stage of preoperative training and in postoperative testing with minimal and $30 \mathrm{~min}$ delay before presentation of pair 5 . ${ }^{*} p<0.025$.

\section{Performance after surgery}

As shown in Figure 7, postoperative performance of both groups was reduced somewhat from the preoperative level. Nevertheless, the rats with hippocampal damage performed as well as control rats when each trial involved presentation of the full sequence of odor choices with minimal memory delay. In contrast, although control rats continued to perform well with the introduction of a 30 min memory delay before the choice on pair 5 , the performance of hippocampal rats was severely impaired. A repeatedmeasures ANOVA comparing performance on preoperative random-order sessions and postoperative sessions with minimal and $30 \mathrm{~min}$ delays confirmed that the performance of the two groups differed across these test phases (group $\times$ phase interaction: $\left.F_{(2,8)}=15.868 ; p=0.0016\right)$. Post hoc analyses revealed that the performance of hippocampal rats differed from that of controls only on testing with the 30 min delay ( $F_{\text {Group at Postop 30-min(1,4) }}=$ $18.02 ; p<0.025)$. The performance of control animals immediately after surgery was marginally decreased with respect to their preoperative level, but this difference did not reach significance $\left(F_{\text {Controls: Preop vs. Postop(1,4) }}=12.333 ; p>0.016 ; \alpha / 3\right.$ was used because three comparisons between means were calculated). In addition, their performance did not significantly decline in subsequent testing with a 30 min delay $\left(F_{\text {Controls: Postop vs. Postop30 } \min (1,4)}=\right.$ $1.4282 ; p>0.016)$. The performance of hippocampal rats did not decline significantly from preoperative to immediate postoperative testing $\left(F_{\text {Hippo: Preop vs. Postop }(1,4)}=1.3016 ; p>0.016\right)$. However, postoperative performance declined significantly with the introduction of the $30 \mathrm{~min}$ delay $\left(F_{\text {Hippo: Postop vs. Postop30 } \min (1,4)}=\right.$ 35.948; $p<0.016)$. Furthermore, the performance of hippocampal rats did not differ from chance on trials with the $30 \mathrm{~min}$ memory delay, whereas the performance of controls on this and other test phases, and that of hippocampal rats on other test phases, was consistently above chance (all $p$-values $<0.05$ ). These findings indicate that rats with radiofrequency lesions are severely impaired in sequence disambiguation that requires remembering early items across a long delay. Furthermore, because the period of postoperative recovery and the amount of postoperative training were similar in the final testing phases of experiments 2 and 3 ( 2 weeks and 10 testing sessions, respectively), it can be con- 
cluded that postoperative sequence training does not necessarily result in recovery of the ability to disambiguate sequences after selective hippocampal damage.

\section{DISCUSSION}

The results of this study show that rats can learn overlapping odor choice sequences and that the hippocampus plays an important role in disambiguation and prediction in sequence memories. Rats with either excitotoxic or radiofrequency lesions of the hippocampus were impaired when required to remember information from early segments of a sequence through an ambiguous component of the sequence. No deficit was apparent when the animals' performance could be based on the immediately preceding, unambiguous information. Although the severity of impairment in sequence alternation was initially equivalent after the two types of lesions, substantial recovery of function was observed selectively in rats with radiofrequency hippocampal lesions. In a version of the task where the sequences were presented widely spaced in random order, animals with radiofrequency hippocampal lesions were not impaired when allowed to perform the sequential choices in immediate succession. However, they were severely impaired when required to remember previous choices within the current sequence across a 30 min delay.

\section{Ibotenate versus radiofrequency lesions}

In recent years many investigators have used fiber-sparing neurotoxic lesions in an effort to produce selective hippocampal lesions that do not eliminate fibers of passage that connect adjacent structures. Because all fibers passing through the hippocampus either originate from or project to one of its subdivisions (Ammon's horn, dentate gyrus, subiculum), the use of neurotoxins may not provide a significant advantage in anatomical selectivity over the use of radiofrequency current. However, because the aberrant activity associated with excitotoxic death likely propagates to other areas, and because the infusion of ibotenate can leak to other areas, there is a danger of subtle widespread damage with this technique.

The present study allows a comparison of the severity and persistence of performance deficits produced by hippocampal lesions of the same size created by the neurotoxin ibotenic acid versus that produced by radiofrequency lesions. Neurotoxic and radiofrequency lesions produced deficits that were similar in magnitude in early postoperative testing, but recovery was observed to a greater extent in animals after radiofrequency lesions. Previous studies comparing ibotenate and conventional cell and fiber damaging lesions of the hippocampus have also reported more severe or more lasting effects of ibotenate than conventional lesions confined to the hippocampus. Jarrard (1986) compared the effects of partial or complete lesions of the hippocampus, produced by either ibotenate or aspiration, on performance in different variations of the radial maze task. He found equivalent deficits after the two types of lesions on spatial memory, in both the working memory and reference memory components of the task. In addition, he observed a severe deficit in working memory with intramaze cues after ibotenate hippocampal lesions, but not with comparable aspiration lesions. No deficit was observed in reference memory for intramaze cues with either type of lesion.

In addition, Anagnostaras et al. (2002) compared the effects of ibotenate and electrolytic lesions of the dorsal hippocampus on fear conditioning. They reported that electrolytic lesions produced a selective deficit in contextual, but not cued, fear conditioning, and the deficits after post-training lesions exhibited a temporally graded retrograde deficit. By contrast, comparable ibotenate lesions resulted in deficits that extended to both the contextual and cued components of the task and produced a flat gradient in the retrograde deficit. After an analysis of the extent of damage several months after the lesions, they concluded that ibotenic acid lesions resulted in widespread cortical cell loss, unlike the effects of electrolytic lesions. The present findings are generally consistent with the findings of Jarrard (1986) and Anagnostaras et al. (2002), in that the deficit after ibotenate lesions observed here can be characterized as more severe in its persistence than that after a comparable lesion made with radiofrequency current.

\section{The nature of the deficit in sequence disambiguation}

One possible explanation for the deficits observed here is that hippocampal damage results in an impairment of olfactory tasks that put a heavy demand on learning and memory capacity. However, other results from our laboratory suggest that rats with selective hippocampal lesions can show fully normal levels of performance on difficult learning tasks and on a task that puts heavy demands on memory capacity. In one study on paired associate learning, normal rats required 300-500 trials to acquire a short list of odor-paired associates (Bunsey and Eichenbaum, 1996). Rats with selective hippocampal lesions were not impaired in acquisition of this task, although there was evidence that the nature of their knowledge about the pairs was abnormal. In another study, the memory capacity of normal rats was strongly challenged when they were required to recognize lists of up to 25 odors (Dudchenko et al., 2000). Nevertheless, rats with selective lesions performed at least as well as normal rats regardless of the memory load. Conversely, in a parallel study (Fortin et al., 2002), we observed that rats with selective hippocampal lesions were impaired in memory for unique (nonambiguous) odor sequences learned in a single trial. These combined results suggest that rats with hippocampal lesions can succeed in very difficult odor learning and memory tasks and can fail in an odor memory task that is easy for normal rats. Thus, task difficulty per se does not explain the deficits observed here. Instead, specific cognitive demands highlighted in sequence disambiguation are implicated as involving hippocampal function.

Comparison of the results from the odor sequence alternation task (experiments 1 and 2) with those from the study on sequence disambiguation involving randomly presented sequences (experiment 3) offers clues about the cognitive demands of sequence memory that require hippocampal function. The stimuli and training protocols of both tasks were very similar; the major differences were in the order of sequence presentation and the addition of a $15 \mathrm{~min}$ intertrial interval in the random-presentation task. One might expect the alternation task to be easier, because cues for the critical choice are available both from the previous trial (make the choice opposite that made at P5 on the preceding trial) and within trials (follow the sequence dictated within the current trial). However, normal rats learned the randompresentation task more readily than they learned the alternation task, suggesting that between-trial cues available in the latter task were not advantageous. Rather, it seems more likely that relatively rapid learning in the random-presentation task was caused by the addition of the $15 \mathrm{~min}$ intertrial interval, which may have substantially reduced proactive interference from preceding trials. Conversely, the high level of proactive interference in the alternation task may have exacerbated the deficit in sequence disambiguation in hippocampal rats, as compared with the ab- 
sence of a deficit observed by hippocampal rats in the initial postoperative performance in the random-presentation task. Nevertheless, when a $30 \mathrm{~min}$ delay was interposed before the critical P5 choice in the random-presentation task, the performance of rats with hippocampal lesions fell to chance. Finally, the absence of a deficit on P6 and the recovery by RF rats on the alternation task suggest that some aspects of sequence memory can be accomplished outside of hippocampal function. Thus, it appears that when memory demands are minimal, as in conditions of low proactive interference or no demand to hold information through ambiguous material, rats with hippocampal damage can succeed in sequence disambiguation. Also, the present results indicate that extended postoperative training can result in improved performance even with a high level of interference. This success may reflect an intact capacity of other brain systems, such as corticalstriatal pathways, to mediate habitual sequences under conditions in which each segment of the sequence rapidly or unambiguously leads to the next (Nissen and Bullemer, 1987; Reber and Squire, 1998). Conversely, when proactive interference is high, or a substantial delay is imposed, a representation mediated by the hippocampus is required to accomplish sequence disambiguation.

\section{Sequence disambiguation and hippocampal neural representations}

Levy (1996) highlighted the capacity of hippocampal circuits in sequence disambiguation for both spatial and nonspatial memory. The present study is the first to our knowledge that directly tests the role of the hippocampus in sequence disambiguation of nonspatial events. There are examples of spatial tasks that can be viewed as highlighting sequence disambiguation, and performance of these tasks is dependent on the hippocampus. One example is T-maze spatial alternation, a task in which rats must remember which of two arms of a T-maze was visited on the previous episode (trial) to select the opposite arm on the current trial. Conceptually, the T-maze alternation task, like the present task, is composed of two alternating episodes that must be distinguished in memory. The separation is rendered more difficult by the fact that the animal must traverse the common stem of the "T" on every trial before completing a left or a right turn on the maze, resulting in a high degree of overlap among places traversed and the same kind of interference that characterizes the present task. Recent electrophysiological data indicate that the hippocampus may mediate performance in the T-maze task by the establishment of distinct representations for sequences of places that are traversed during execution of the two types of trials. These studies show that different populations of hippocampal neurons distinctly encode the ambiguous components of the left-turn and right-turn episodes in this task (Frank et al., 2000; Wood et al., 2000). The present results suggest that the capacity of the hippocampus to form distinct representations for overlapping sequences extends to nonspatial memories as well. This type of processing is seen as critical to distinguishing and linking related memories in an overall organization that supports the capacity for declarative memory (Eichenbaum et al., 1999).

\section{REFERENCES}

Anagnostaras SG, Gale GD, Fanselow MS (2002) The hippocampus and Pavlovian fear conditioning: reply to Bast et al. Hippocampus, in press.

Bunsey M, Eichenbaum H (1996) Conservation of hippocampal memory function in rats and humans. Nature 379:255-257.

Chiba AA, Kesner RP, Reynolds AM (1994) Memory for spatial location as a function of temporal lag in rats: role of hippocampus and medial prefrontal cortex. Behav Neural Biol 61:123-131.

Clayton NS, Dickinson A (1998) Episodic-like memory during cache recovery by scrub jays. Nature 395:272-274.

Dudchenko PA, Wood ER, Eichenbaum H (2000) Neurotoxic hippocampal lesions have no effect on odor span and little effect on odor recognition memory, but produce significant impairments on spatial span, recognition, and alternation. J Neurosci 20:2964-2977.

Eichenbaum H, Dudchenko P, Wood E, Shapiro M, Tanila H (1999) The hippocampus, memory, and place cells: is it spatial memory or memory space? Neuron 23:209-226.

Fortin NJ, Agster KL, Eichenbaum HB (2002) Critical role of the hippocampus in memory for sequences of events. Nat Neurosci 5:458-462.

Frank LM, Brown EN, Wilson M (2000) Trajectory encoding in the hippocampus and entorhinal cortex. Neuron 27:169-178.

Gaffan D (1994). Scene-specific memory for objects: a model of episodic memory impairment in monkeys with fornix transection. J Cognit Neurosci 6:305-320.

Girden ER (1992) ANOVA: Repeated-measures. In: Sage University paper series on quantitative applications in the social sciences, 07-084. Newbury Park, CA: Sage.

Jarrard LE (1986) Selective hippocampal lesions and behavior: implications for current research and theorizing. In: The hippocampus, Vol 4 (Isaacson RL, Pribram KH, eds), pp 93-126. New York: Plenum.

Kesner RP, Novak JM (1982) Serial position curve in rats: role of the dorsal hippocampus. Science 218:173-175.

Kirk RE (1982) Experimental design: procedures for the behavioral sciences. Belmont, CA: Brooks/Cole.

Levy WB (1996) A sequence predicting CA3 is a flexible associator that learns and uses context to solve hippocampal-like tasks. Hippocampus 6:579-590.

Lisman JE (1999) Relating hippocampal circuitry to function: recall of memory sequences by reciprocal dentate-CA3 interactions. Neuron 22:233-242.

Mishkin M, Suzuki WA, Gadian DG, Vargha-Khadem F (1997) Hierarchical organization of cognitive memory. Philos Trans R Soc Lond B Biol Sci 352:1461-1467.

Nissen MJ, Bullemer P (1987) Attentional requirements of learning: evidence from performance measures. Cognit Psychol 19:1-32.

O'Keefe J, Nadel L (1978) The hippocampus as a cognitive map. New York: Oxford UP.

Reber PJ, Squire LR (1998) Encapsulation of implicit and explicit memory in sequence learning. J Cognit Neurosci 10:248-263.

Sohal VS, Hasselmo ME (1998) Changes in GABAB modulation during a theta cycle may be analogous to the fall of temperature during annealing. Neural Comput 10:889-902.

Swanson LW (1992) Brain maps: structure of the rat brain. Amsterdam: Elsevier.

Vargha-Khadem F, Gadian DG, Watkins KE, Connelly A, Van Paesschen W, Mishkin M (1997) Differential effects of early hippocampa pathology on episodic and semantic memory. Science 277:376-380.

Wallenstein GV, Eichenbaum H, Hasselmo ME (1998) The hippocampus as an associator of discontiguous events. Trends Neurosci 21:317-323.

Wood ER, Dudchenko PA, Robitsek RJ, Eichenbaum HB (2000) Hippocampal neurons encode information about different types of memory episodes occurring in the same location. Neuron 27:623-633.

Zola-Morgan S, Squire LR, Clower RP, Rempel NL (1993) Damage to the perirhinal cortex exacerbates memory impairment following lesions to the hippocampal formation. J Neurosci 13:251-265.

Zola-Morgan S, Squire LR, Ramus SJ (1994) Severity of memory impairment in monkeys as a function of locus and extent of damage within the medial temporal lobe memory system. Hippocampus 4:483-495. 